OPEN ACCESS

Edited by:

Lino Nobili,

University of Genoa, Italy

Reviewed by:

Arturo Garay,

Centro de Educación Médica e Investigaciones Clínicas Norberto

Quirno (CEMIC), Argentina

Jupi Li,

China Medical University, Taiwan

*Correspondence:

Chia-Chou Yeh

yehcc0530@gmail.com

Tzung-Yi Tsai

dm732024@tzuchi.com.tw

How-Ran Guo

hrguo@ncku.edu.tw

tThese authors have contributed equally to this work

Specialty section:

This article was submitted to

Sleep Disorders,

a section of the journal

Frontiers in Neurology

Received: 19 July 2020 Accepted: 10 December 2020

Published: 20 January 2021

Citation:

Chiao Y-W, Livneh $H$, Guo $H-R$,

Chen W-J, Lu M-C, Lin M-C, Yeh C-C and Tsai T-Y (2021) Use of Chinese

Herbal Medicines Is Related to a Reduction in Depression Risk Among Patients With Insomnia: A Matched Cohort Study.

Front. Neurol. 11:583485. doi: 10.3389/fneur.2020.583485

\section{Use of Chinese Herbal Medicines Is Related to a Reduction in Depression Risk Among Patients With Insomnia: A Matched Cohort Study}

\author{
Yun-Wen Chiao ${ }^{1,2 t}$, Hanoch Livneh ${ }^{3 t}$, How-Ran Guo ${ }^{4,5,6 *}$, Wei-Jen Chen ${ }^{1 \dagger}$, Ming-Chi Lu ${ }^{7,8}$, \\ Miao-Chiu Lin ${ }^{9 t}$, Chia-Chou Yeh ${ }^{1,10 *}$ and Tzung-Yi Tsai ${ }^{6,11,12 *}$
}

${ }^{1}$ Department of Chinese Medicine, Dalin Tzuchi Hospital, The Buddhist Tzuchi Medical Foundation, Chiayi, Taiwan, ${ }^{2}$ Department of Traditional Medicine, Kaohsiung Veterans General Hospital, Kaohsiung, Taiwan, ${ }^{3}$ Rehabilitation Counseling Program, Portland State University, Portland, OR, United States, ${ }^{4}$ Department of Occupational and Environmental Medicine, National Cheng Kung University Hospital, Tainan, Taiwan, ${ }^{5}$ Occupational Safety, Health, and Medicine Research Center, National Cheng Kung University, Tainan, Taiwan, ${ }^{6}$ Department of Environmental and Occupational Health, College of Medicine, National Cheng Kung University, Tainan, Taiwan, ${ }^{7}$ Division of Allergy, Immunology and Rheumatology, Dalin Tzuchi Hospital, The Buddhist Tzuchi Medical Foundation, Chiayi, Taiwan, ${ }^{8}$ School of Medicine, Tzu Chi University, Hualien, Taiwan, ${ }^{9}$ Department of Nursing, Dalin Tzuchi Hospital, The Buddhist Tzuchi Medical Foundation, Chiayi, Taiwan, ${ }^{10}$ School of Post-Baccalaureate Chinese Medicine, Tzu Chi University, Hualien, Taiwan, ${ }^{11}$ Department of Medical Research, Dalin Tzuchi Hospital, The Buddhist Tzuchi Medical Foundation, Chiayi, Taiwan, ${ }^{12}$ Department of Nursing, Tzu Chi University of Science and Technology, Hualien, Taiwan

Objective: Subjects with insomnia have a higher risk of depression, thus possibly making them live with serious health conditions. To date, information regarding the effect of Chinese herbal medicines (CHMs), a commonly used complementary and alternative medicine, on depression risk among people with insomnia is still unknown. This study aimed to investigate the effect of $\mathrm{CHMs}$ on the risk of depression among individuals with insomnia.

Methods: This cohort study used a national health insurance database to identify 68,573 subjects newly diagnosed with insomnia, aged 20-70 years, who received treatment between 1998 and 2010. Using propensity score matching, we randomly selected 26,743 CHMs users and 26,743 non-CHMs users from this sample. All enrollees were followed to the end of 2012 to identify any treatment for depression as the end point. Cox proportional hazards regression was used to compute the adjusted hazard ratio of depression associated with CHMs use.

Results: After utilizing the propensity score matching, we randomly selected 26,743 CHMs users and 26,743 non-CHMs users from this sample. During follow up, 3,328 CHMs users and 6,988 non-CHMs users developed depression at incidence rates of 17.24 and 37.97 per 1,000 person-years, respectively. CHMs users had a lower depression risk than the non-CHMs users (adjusted hazard ratio $=0.44 ;$ 95\% Confidence Interval, 0.42-0.46). The greatest effect was observed for those taking $\mathrm{CHMs}$ for more than 2 years. Gegen, Huangqin, Dan-Shen, Beimu, Dahuang, Shegan, Shu-jing-huo-xue-tang, Ge-gen-tang, Shao-yao-gan-cao-tang and 
Píng wèi săn were significantly associated with a lower risk of depression.

Conclusions: Findings from this study demonstrated that adding CHMs to conventional treatment significantly reduces depression risk among patients with insomnia.

Keywords: insomnia, Chinese herbal medicine, depression, complementary and alternative, cohort study

\section{KEY POINTS}

- This is the first investigation to explore the effects of CHMs use against the depression onset among individuals with insomnia.

- We discovered that the CHMs use was associated with a lower risk of depression after adjustment for the potential confounding factors.

- The more predominant effect was observed among those receiving CHMs for more than 2 years, with up to $80 \%$ lower risk of depression.

- In addition to the positive effect of CHMs, the findings of this study further indicated the commonly prescribed herbal products that may be related to reduced depression risk, thus paving the way for further pharmacological investigations.

\section{INTRODUCTION}

Insomnia is a common sleep disorder affecting about one third of the general population worldwide. It is characterized by difficulty in falling asleep, difficulty in staying asleep, and sleep that is non-restorative in nature (1). Compared to the general population, those with persistent insomnia have a $58 \%$ increased risk of all-cause mortality (2). The rise in incidence of chronic insomnia not only triggers major health challenges but also results in tremendous economic loss. The total cost associated with insomnia is estimated at \$92.5-107.5 billion annually in the US alone (3), which implied that insomnia was clearly an important public health concern requiring particular attention.

Notably, insomnia would further result in sleepiness, fatigue or cognitive impairment, thus pre-disposing individuals who experience insomnia to developing depression. A meta-analysis of 21 longitudinal studies demonstrated that insomnia may double the risk of depression independently of conventional risk factors (4). At its worst, a diagnosis of depression increases the risk of death by as much as $50 \%$ (5). Moved by the tragic case presented above, interventions geared at preventing or lessening the susceptibility of depression may be of utmost importance.

In Asian cultures, Chinese herbal medicines (CHMs) are complementary and alternative medical therapies commonly used for patients with chronic diseases. Several of these herbs have been proven to improve cognitive function by suppressing monoamine oxidase activity and oxidative stress, upregulating neurotrophins and modulating the function of the hypothalamic-pituitary-adrenal (HPA) axis in patients with neurodegenerative diseases (6). Previous research has illustrated that CHMs produce therapeutic effects of equal magnitude to hypnosis through multiple bioactivity channels in the central neural system $(7,8)$. One systematic review affirmed the efficacy and safety of CHMs to be as effective as that of antidepressants
(9). Another pharmacological research summarized the antidepressant mechanisms of active components, such as elevated synaptic concentrations of monoamines, modifying the HPA axis dysfunctions, and increasing synaptic plasticity (10). Such therapeutic benefits bolster the potential of CHMs use for treating depression in patients with insomnia.

Considering the potential association of insomnia with a subsequent risk of depression and the limited information on the capacity of CHMs to modify this association, we believe findings from a long-term population-based cohort study may be of importance in allocating medical resources and instituting evidence-based policymaking. Thus, we examined a nationwide population-based database to compare the risk of depression among patients with insomnia who did and did not receive CHMs.

\section{METHODS}

\section{Data Source}

The present cohort study analyzed data from the Longitudinal Health Insurance Database (LHID 2000), which is managed by the National Health Insurance (NHI) Administration. Taiwan launched a single-payer NHI program in 1995 to remove financial barriers to medical care for all legal residents. As of 2017, over $99 \%$ of Taiwan's population had enrolled in this program. The LHID 2000 is based on a sub-set of the NHI program made up of one million randomly sampled people who were traced retrospectively from 1996 to 2012. These one million insured individuals did not differ significantly in sex or age from the general population, as a multistage stratified systematic sampling method was used (11). This database contains all NHI enrollment files, claims data and data from a prescription drugs registry, providing comprehensive utilization information on the subjects covered by the insurance program.

\section{Ethics}

The current investigation used retrospective data from an administrative database in which patients were made anonymous to the researchers. It was also conducted in accordance with the Helsinki Declaration and was approved by the Institutional Review Board and Ethics Committee of Buddhist Dalin Tzu Chi Hospital, Taiwan (No. B10004021-3).

\section{Study Participants}

The methods used to select the study subjects are illustrated in Figure 1. Diagnoses in the insurance claims data were coded using the International Classification of Disease, 9th Revision, Clinical Modification (ICD-9-CM). Patients who were older than 20 years, and were newly diagnosed with insomnia between 1998 


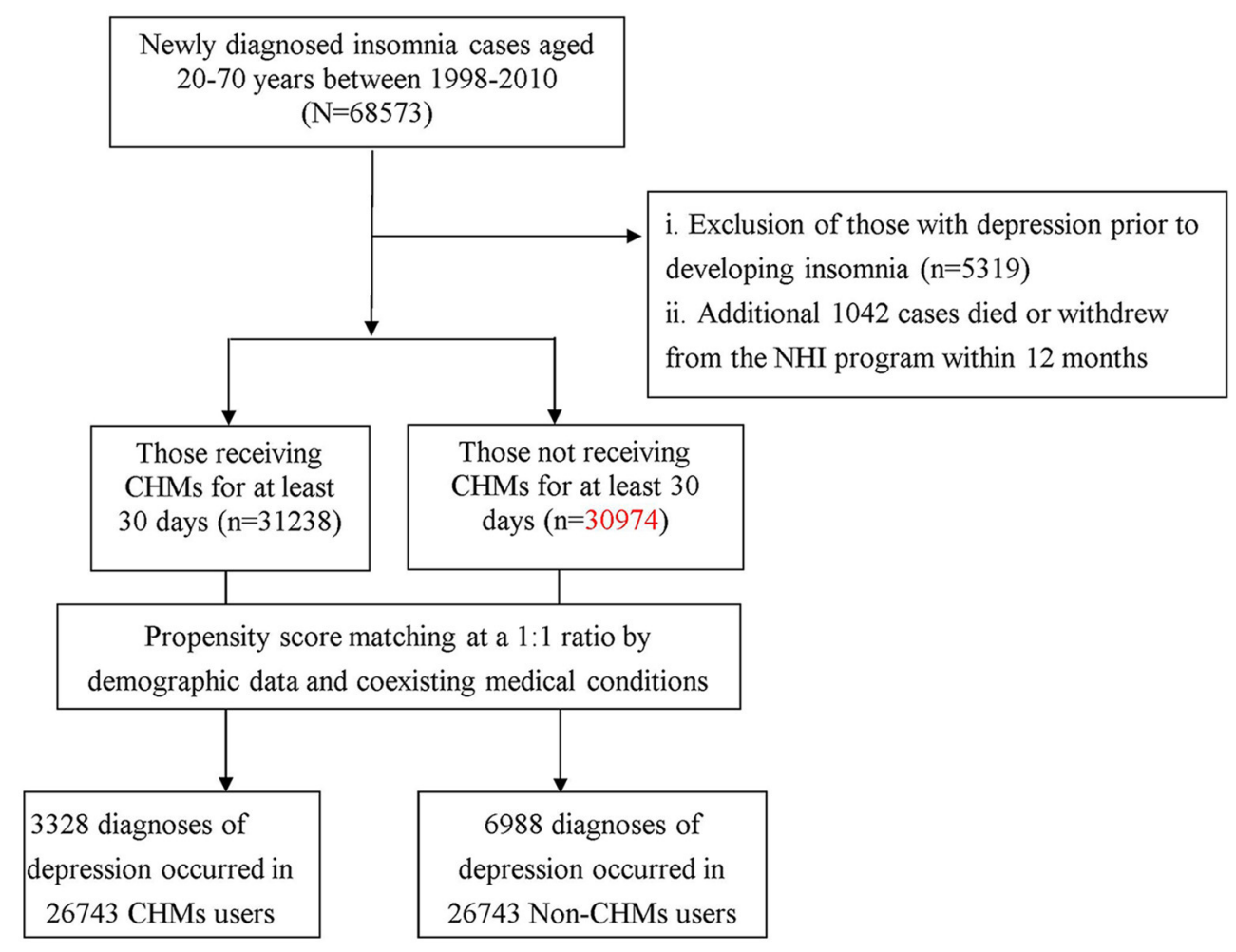

FIGURE 1 | Flow chart of selection and follow-up of study subjects.

and 2010 (ICD-9-CM codes: 307.41, 307.42, 780.50, 780.52), were considered for enrollment into the study.

To reduce the potential for disease misclassification, we selected patients had diagnoses of insomnia in at least three outpatient visits, or were admitted to the hospital with a primary diagnosis of insomnia within the observation period $(n=$ 68,573). To establish a temporal link between insomnia and depression, we excluded those who were followed for $<1$ year after the onset of insomnia $(n=1,042)$ and those who had a prior diagnosis of depression before the first insomnia diagnosis $(n=5,319)$. Patients were identified as having depression if they had at least three ambulatory visits for treatment or had been hospitalized due to depression, as reflected in the use of ICD-9CM codes 296.2, 296.3,300.4, or 311. A group of 62,212 subjects with insomnia were included in the final data analysis.

As only certified Chinese medicine physicians are permitted to prescribe CHMs in Taiwan. We, therefore, used the frequency of visits to Chinese medicine physicians to verify the CHMs exposure of each insomniac patient. Those receiving CHMs to treat insomnia for more than 30 days were deemed CHMs users, whereas those treated for 30 days or less were considered nonCHMs users $(12,13)$. Based on this procedure, 31,238 cases were designated as CHMs users. A comparison cohort was randomly selected from the remaining insured insomnia cases without CHMs use. For each patient receiving CHMs treatment, one control without CHMs was selected by 1:1 matching based on a propensity score. The propensity score was calculated using logistic regression based on patient demographics and baseline comorbidities at enrollment. To consider the immortal time bias (14), we defined the index date for the follow-up period for the patients with insomnia who were classified as non-CHMs users to be the date of the first insomnia diagnosis. The index date for the follow-up period for the patients with insomnia who used CHMs was the first date of the initiation of CHMs treatment. Each patient with insomnia was followed from the index date until 31 December 2012, death or being censored. The follow-up time, in person-years (PYs), was calculated for each insomniac patient until the diagnosis of depression, death or until being censored due to withdrawal from the insurance system or loss to follow-up.

\section{Covariates Assessment}

Covariates consisted of the baseline sociodemographic characteristics and comorbidities. Sociodemographic characteristics considered in this study included age, gender, income (for estimating insurance payments) and urbanization level of the registered resident region. Monthly income was defined as the subject's own insurable wage and stratified into three levels: $\leq 17,880$ New Taiwan Dollar (NTD) (low); 17,88143,900 NTD (median); and $\geq 43,901$ NTD (high). In addition, we also grouped the resident region by collapsing each geographic location into one of three strata, namely, urban (levels 1-2), suburban (levels 3-4) and rural (levels 5-7), with higher levels indicating greater urbanization (15). Baseline comorbidities for each subject were determined by individual medical records in the year preceding cohort entry, all of which were assessed by 
the established Charlson-Deyo comorbidity index (CCI) (16). The CCI score consisted of 17 chronic diseases, each with a score of 1-to- 6 points. The sum of these scores was regarded as a continuous variable for the burden of comorbidities, with higher scores indicating a more severe impact from the these comorbidities.

\section{Statistical Analysis}

The SAS version 9.3 software (SAS Institute Inc, Cary, NC, USA) was used for data analysis. Distributions of socio-demographic data and comorbidities between the enrollees who did and did not receive CHMs were compared by the chi-square test and independent Student's $t$-test. Cox proportional hazards regression analysis was applied to compute the adjusted hazard ratio (HR) with its $95 \%$ confidence interval (CI) of the risk of depression in association with CHMs use, after accounting for the confounders reported at baseline. To further test the robustness of the relationship between CHMs use and depression risk, we divided the CHMs users into three subgroups: those using CHMs for 31-365 days, for 366-730 days and for more than 730 days. The Cox proportional hazards regression analysis with Bonferroni correction was applied for multiple comparisons among the groups. We also used the Kaplan-Meier method to estimate the cumulative risk of depression for different groups and tested the difference with the log-rank test. Furthermore, a stratified analysis by age and sex using Cox proportional hazards regression was conducted to assess the relative risk of depression associated with CHMs. The proportional hazards assumption was examined by plotting the $\log [-\log$ (survival function) $]$ vs. the $\log$ of survival time. Differences of $p<0.05$ were determined to be statistically significant.

\section{RESULTS}

The CHMs user and non-CHMs user cohorts each provided data for 26,743 patients. Table 1 shows the pertinent characteristics of the two groups, including the distribution of age, sex, monthly income, residential area and comorbidities, and indicating that the two groups were comparable on these characteristics. The mean age of the CHMs users and non-CHMs users was 48.5 \pm 12.3 and $48.5 \pm 12.5$ years, respectively. Female patients predominated in both groups, at $64.0 \%$. The majority of participants had monthly income levels of 17,881-43,900 NTD (48.7\%) and resided in more urbanized areas (57.8\%).

Among the 53,486 patients with insomnia and no history of prior depression, who were enrolled in this study, 10,316 first episodes of depression were identified; 6,988 were reported among the non-CHMs users and 3,328 among the CHMs users during follow-up periods of 184,029.27 and 193,037.73 PYs, respectively. The incidence rate of depression was lower among CHMs users than in non-CHMs users (17.24 vs. 37.97, respectively, per 1000 PYs), with the adjusted HR of 0.44 (95\% CI $=0.42-0.46)$ (Table 2). Table 2 also lists the crude and adjusted HRs with Bonferroni correction using the non-CHMs users as reference. Those who used CHMs treatment for more than 730 days had an $80 \%$ lower risk of depression than non-CHMs users (adjusted HR $=0.20$;
TABLE 1 | Demographic data and selected comorbidities of the study subjects.

\begin{tabular}{|c|c|c|c|c|}
\hline \multirow[t]{2}{*}{ Variables } & \multirow[t]{2}{*}{ All } & \multirow{2}{*}{$\frac{\text { Non-CHMs users }}{n=26,743(\%)}$} & \multirow{2}{*}{$\frac{\text { CHMs users }}{n=26,743(\%)}$} & \multirow[t]{2}{*}{$p$-value* } \\
\hline & & & & \\
\hline Age (yr) & & & & 0.44 \\
\hline$\leq 50$ & $28,771(53.8)$ & 14,341 (53.6) & $14,430(54.0)$ & \\
\hline$>50$ & $24,715(46.2)$ & $12,402(46.4)$ & $12,313(46.0)$ & \\
\hline Mean (SD) & $48.5 \pm 12.4$ & $48.5 \pm 12.3$ & $48.5 \pm 12.5$ & 0.97 \\
\hline Gender & & & & 0.69 \\
\hline Female & $34,224(64.0)$ & $17,090(63.9)$ & $17,134(64.1)$ & \\
\hline Male & $19,262(36.0)$ & $9,653(36.1)$ & $9,609(35.9)$ & \\
\hline Monthly income & & & & 0.40 \\
\hline Low & $24,988(46.7)$ & $12,417(46.4)$ & $12,571(47.0)$ & \\
\hline Median & $26,037(48.7)$ & 13,095 (49.0) & $12,942(48.4)$ & \\
\hline High & $2,461(4.6)$ & $1,231(4.6)$ & $1,230(4.6)$ & \\
\hline Residential area & & & & 0.96 \\
\hline Urban & $30,911(57.8)$ & $15,454(57.8)$ & $15,457(57.8)$ & \\
\hline Suburban & $8,420(15.7)$ & 4,221 (15.8) & $4,199(15.7)$ & \\
\hline Rural & $14,155(26.5)$ & 7,068 (26.4) & 7,087 (26.5) & \\
\hline $\mathrm{CCl}$ & & & & 0.28 \\
\hline Mean (SD) & $4.0(6.7)$ & $3.9(6.6)$ & $4.0(6.8)$ & \\
\hline
\end{tabular}

CHMs, Chinese herbal medicines; SD: standard deviation; CCI, Charlson-Deyo comorbidity index.

*All p-values were derived from chi-square test, except for $\mathrm{CCl}$ that was calculated with independent Student's t-test.

95\% CI $=0.18-0.23$. Figure 2 presents the Kaplan-Meier estimates of the cumulative incidence of depression for the four groups during the 15 -year follow-up period, after adjusting for patients' age, sex, CCI scores, monthly incomes and urbanization levels. The cumulative incidence of depression of those receiving CHMs treatments for more than 730 days was significantly lower than for those not receiving CHMs (log-rank test, $p<0.001)$.

We performed an additional stratified analysis by age and sex to determine the effect of $\mathrm{CHMs}$ on risk of depression (Table 3). Findings showed that both female and male patients with insomnia, who also received CHMs, had a lower risk of depression than did their non-CHMs counterparts, with an adjusted HR of 0.41 $(95 \% \mathrm{CI}=0.38-0.43)$ and $0.53(95 \% \mathrm{CI}=0.49-0.57)$, respectively. A more remarkable beneficial effect of CHMs was observed among younger subjects. In females, decreases in adjusted HR were greater for CHMs users $\leq 50$ years of age (adjusted HR $=0.32,95 \% \mathrm{CI}=0.31-0.35$ ); in males, the effect of CHMs on the risk of depression was greater for those $\leq 50$ years of age, with an adjusted HR of 0.45 $(95 \% \mathrm{CI}=0.41-0.50)$.

The most commonly prescribed Chinese herbal products for insomnia patients are summarized in Table 4. Of these, 10 were found to substantially lessen the subsequent risk of depression: Ge Gen (Pueraria lobate), Huang Qin (Scutellaria Baicale), Dan Shen (Salvia miltiorrhiza), Bei Mu (Fritillariae Thunbergii), Da Huang (Rheum palmatum), She Gan (Belamcanda chinensis), 
TABLE 2 | Risk of depression for insomnia subjects with and without CHMs.

\begin{tabular}{|c|c|c|c|c|c|}
\hline Patient group & Event & PYs & Incidence (per 1000 PYs) & Crude HR (95\% CI) & Adjusted HR* $(95 \% \mathrm{Cl})$ \\
\hline Non-CHMs users & 6,988 & 184029.27 & 37.97 & 1 & 1 \\
\hline CHMs users & 3,328 & 193037.73 & 17.24 & $0.45(0.43-0.47)$ & $0.44(0.42-0.46)$ \\
\hline CHMs use within $30-180$ days & 2,352 & 107147.47 & 21.95 & $0.58(0.53-0.60)$ & $0.55(0.53-0.58)$ \\
\hline CHMs use within $181-365$ days & 527 & 38744.11 & 13.60 & $0.36(0.33-0.39)$ & $0.34(0.31-0.37)$ \\
\hline CHMs use within 366 to 730 days & 311 & 28278.19 & 11.00 & $0.29(0.26-0.33)$ & $0.28(0.25-0.31)$ \\
\hline CHMs use for more than 730 days & 138 & 18867.96 & 7.31 & $0.20(0.17-0.23)$ & $0.20(0.18-0.23)$ \\
\hline
\end{tabular}

*Model adjusted for age, gender, urbanization level, monthly income, medication usage and CCl score.

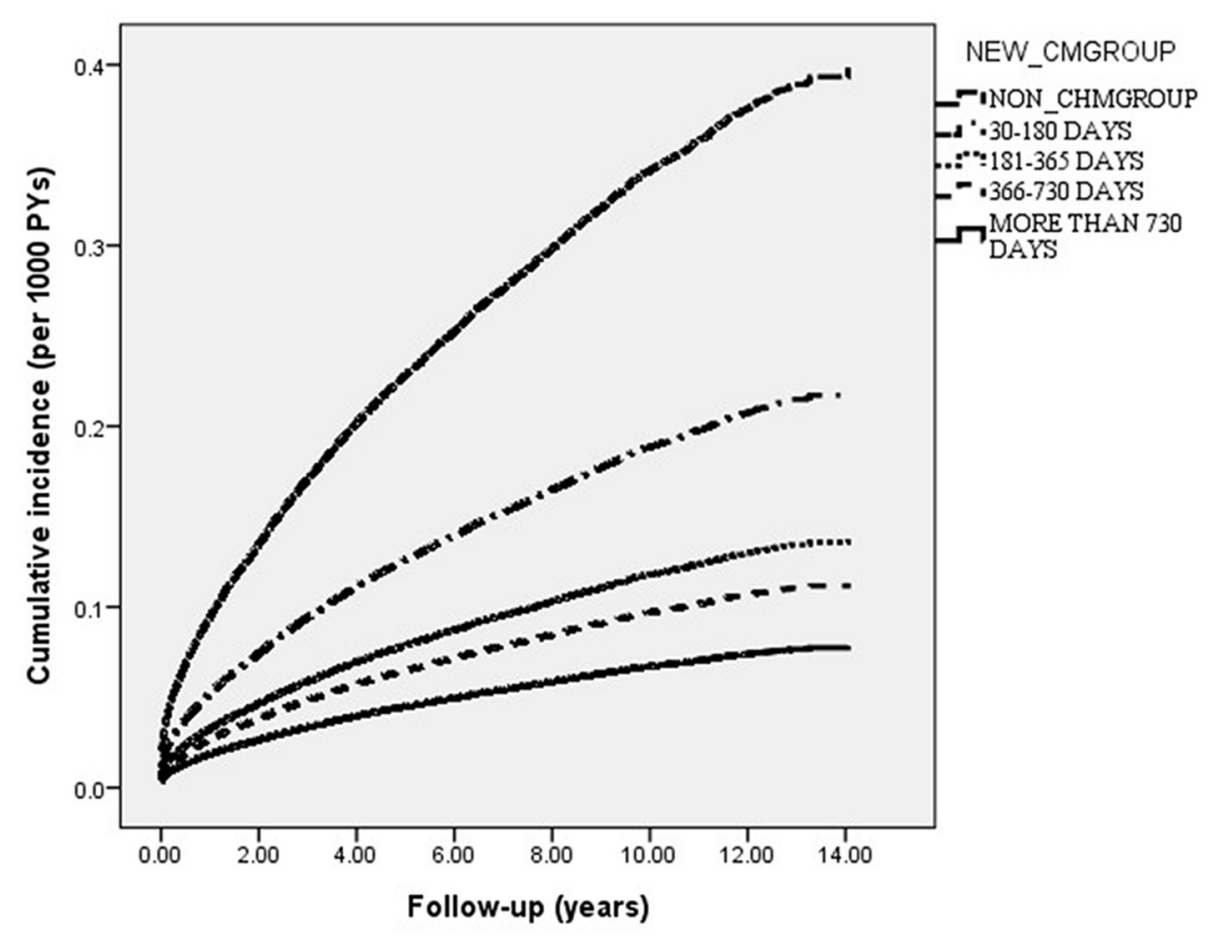

FIGURE 2 | Cumulative incidence of depression in insomnia patients with and without receiving CHMs treatment during the 15-year study period (Log-rank test, $P<0.001)$

TABLE 3 | Incidence (per 1000 PYs) and depression risk for insomnia patients with and without CHMs in the stratification of sex and age.

\begin{tabular}{|c|c|c|c|c|c|c|c|c|}
\hline \multirow[t]{2}{*}{ Variables } & \multicolumn{3}{|c|}{ Non-CHMs users } & \multicolumn{3}{|c|}{ CHMs users } & \multirow[t]{2}{*}{ Crude HR (95\% Cl) } & \multirow[t]{2}{*}{ Adjusted HR (95\% Cl) } \\
\hline & Case & PYs & Incidence & Case & PYs & Incidence & & \\
\hline \multicolumn{9}{|l|}{ Female } \\
\hline$\leq 50$ & 2,949 & 52986.41 & 55.66 & 1,158 & 64092.24 & 18.07 & $0.32(0.30-0.35)$ & $0.32(0.31-0.35)^{*}$ \\
\hline$>50$ & 2,026 & 60012.79 & 33.76 & 1,106 & 59922.36 & 18.46 & $0.55(0.49-0.57)$ & $0.53(0.50-0.57)^{*}$ \\
\hline All & 4,975 & 112999.2 & 44.03 & 2,264 & 124014.6 & 18.26 & $0.41(0.42-0.46)$ & $0.41(0.38-0.43)^{\Omega}$ \\
\hline \multicolumn{9}{|l|}{ Male } \\
\hline$\leq 50$ & 1,296 & 39882.51 & 32.50 & 590 & 39102.37 & 15.09 & $0.46(0.41-0.50)$ & $0.45(0.41-0.50)^{*}$ \\
\hline$>50$ & 717 & 31207.56 & 22.98 & 474 & 29920.76 & 15.84 & $0.69(0.59-0.75)$ & $0.66(0.59-0.74)^{*}$ \\
\hline All & 2,013 & 71090.07 & 28.32 & 1,064 & 69023.13 & 15.42 & $0.54(0.49-0.57)$ & $0.53(0.49-0.57)^{\Omega}$ \\
\hline
\end{tabular}

* Model adjusted for urbanization level, monthly income, medication usage and CCl score.

${ }^{\Omega}$ Model adjusted for age, urbanization level, monthly income, medication usage and CCl score. 
TABLE 4 | Risk of depression in relation to the 10 most-used single-herb and multi-herb products for insomnia patients.

\begin{tabular}{|c|c|c|c|}
\hline Chinese herbal name & Number of prescriptions & Crude HR $(95 \% \mathrm{Cl})$ & Adjusted HR * $(95 \% \mathrm{Cl})$ \\
\hline \multicolumn{4}{|l|}{ Single-herb products } \\
\hline Yan-Hu-Suo & 11,868 & $0.81(0.68-1.09)$ & $0.84(0.69-1.11)$ \\
\hline Gegen & 11,549 & $0.46(0.43-0.49)$ & $0.44(0.42-0.46)$ \\
\hline Huangqin & 10,291 & $0.46(0.44-0.49)$ & $0.44(0.42-0.47)$ \\
\hline Multiflora & 9,803 & $0.86(0.72-1.10)$ & $0.88(0.73-1.09)$ \\
\hline Dan-Shen & 9,234 & $0.47(0.44-0.46)$ & $0.46(0.43-0.49)$ \\
\hline Beimu & 9,116 & $0.43(0.41-0.46)$ & $0.42(0.39-0.45)$ \\
\hline Dahuang & 6,748 & $0.49(0.45-0.52)$ & $0.45(0.42-0.48)$ \\
\hline Suan Zao Ren & 6,379 & $0.87(0.73-1.06)$ & $0.85(0.73-1.11)$ \\
\hline Hai Piao Xiao & 6,051 & $0.85(0.64-1.08)$ & $0.84(0.63-1.09)$ \\
\hline Shegan & 4,527 & $0.44(0.40-0.48)$ & $0.42(0.38-0.46)$ \\
\hline \multicolumn{4}{|l|}{ Multi-herb products } \\
\hline Jia-Wei-Xiao-Yao-San & 14,573 & $0.89(0.73-1.09)$ & $0.88(0.74-1.05)$ \\
\hline Shu-Jing-Huo-Xue-Tang & 12,684 & $0.45(0.42-0.47)$ & $0.45(0.42-0.48)$ \\
\hline Ge-Gen-Tang & 11,549 & $0.46(0.43-0.49)$ & $0.45(0.42-0.48)$ \\
\hline Shao-Yao-Gan-Cao-Tang & 11,212 & $0.43(0.41-0.46)$ & $0.43(0.40-0.45)$ \\
\hline Chuan Xiong Cha Tiao San & 9,162 & $0.87(0.75-1.07)$ & $0.85(0.72-1.06)$ \\
\hline Píng wèi săn & 7,931 & $0.46(0.43-0.49)$ & $0.45(0.42-0.47)$ \\
\hline Suan Zao Ren Tang & 7,883 & $0.86(0.72-1.08)$ & $0.89(0.74-1.07)$ \\
\hline Tian Wang Bu Xin Dan & 7,706 & $0.88(0.74-1.07)$ & $0.89(0.74-1.10)$ \\
\hline Ban Xia Xie Xin Tang & 7,566 & $0.89(0.76-1.08)$ & $0.88(0.74-1.11)$ \\
\hline Xiao Chai Hu Tang & 6,752 & $0.89(0.78-1.13)$ & $0.89(0.76-1.14)$ \\
\hline
\end{tabular}

${ }^{\star}$ Model adjusted for age, sex, urbanization level, monthly income, and comorbidities.

Shu-jing-huo-xue-tang (SJHXT), Ge-gen-tang (GGT), Shao-yaogan-cao-tang (SYGCT) and Ping-wei-san (PWS).

\section{DISCUSSION}

This is the first large scale, retrospective cohort study exploring the risk of depression in patients with insomnia who either received or did not receive CHMs treatment. Although several studies were reported on the benefit of using CHMs for sleep disorders, or for other psychological problems (17-19), no studies have yet explored the relationship of CHMs with the risk of depression among those with sleep disorders.

The findings of the present study are consistent with those of prior epidemiological studies, showing that insomnia usually occurs before the first instance of depression (20). Our study lends initial support for integrating CHMs into conventional therapy, and suggests that such practice may reduce the risk of depression by as much as $60 \%$. The dose-response relationship reported herein could further clarify the causality between CHMs use and a reduced susceptibility to develop depression. Our results indicate that the extracts of some Chinese herbal products may suppress the production of inflammatory cytokines and modulate the neurotransmitter imbalance that results in depression.

For example, the commonly prescribed multi-herb formula SJHXT was found to efficiently diminish the risk of depression in patients with insomnia. Using a rodent model, Shu and colleagues discovered that SJHXT could modulate the activity of the alpha-2 adrenoceptor ( $\alpha 2-\mathrm{AR})$ (21). A subsequent study indicated that depressive disorders appeared to be associated with increased $\alpha 2 \mathrm{AR}$ sensitivity and responsiveness (22). The dysregulation of the $\alpha 2$-AR pathway may contribute to the release of pro-inflammatory cytokines, such as tumor necrosis factor$\alpha$ (TNF- $\alpha$ ), interleukin-1 (IL-1) and IL-6, thus increasing the susceptibility to depression (23).

The current study indicated that the use of SYGCT could lessen the risk of depression for those with insomnia. We speculate that the conspicuous anti-depressant effect of SYGCT may be related to its ingredients, including Paeonia lactiflora Pall. and Glycyrrhiza uralensis. A recent study showed that $P$. lactiflora Pall., a major compound purified from this formula, can significantly inhibit the hyperfunction of the HPA axis (24). Meanwhile, G. uralensis works by promoting the release of 5-hydroxytryptamine and increasing synaptic plasticity in the hippocampus (10), thereby allowing neurotransmitters to function more efficiently.

Another herbal product proven effective in lessening depression risk is PWS. An earlier report suggests that Magnolia officinalis, a major component in PWS, can inhibit neuroinflammation and oxidative stress in the prefrontal cortex and improve the levels of brain-derived neurotrophic factor (BDNF) protein in the hippocampus, as shown in a rodent model of depressive disorder (25). We also identified the positive therapeutic effects of GGT, as well as of Ge Gen. 
We speculate that puerarin, a major isoflavone glycoside purified from these agents, may increase cerebral blood flow via multiple neuroprotective effects, including anti-apoptosis and anti-inflammatory effects, and reduce oxidative stress (26), which may point to the possible mechanisms responsible for the positive effects of these formulae.

With respect to other commonly used single-herb products, we observed that the use of Dan-Shen was related to a reduced risk of depression onset. This remedy has been shown to protect mice from $A \beta$-induced neurotoxicity by inhibiting increases in TNF- $\alpha$, IL-6 or TNF- $\alpha$ levels $(27,28)$. These mediators are well-known to play important roles in the pathogenesis of neuropsychiatric symptoms, especially depression (29).

Additionally, Huang-Qin, Da-Huang, Bei-Mu and She-Gan are believed to exert their neuroprotection effect by suppressing pivotal activities of nuclear factor kappa-light-chain-enhancer of activated B cells associated with inflammatory pathways and cell apoptosis (30-34). Furthermore, the baicalin in HuangQin and emodin in Da-Huang can mediate BDNF expression, ameliorating depressive-like behavior $(35,36)$. BDNF in brain areas related to emotions, such as the hippocampus and cortex, can support the survival of neurons, as well as the differentiation and growth of new synapses $(37,38)$. Finally, it has been argued that tectorigenin, an isoflavonoid found in She-Gan, has a confirmed neuroprotective effect against cytotoxicity and apoptosis $(39,40)$.

Findings regarding the positive therapeutic effect of herbal formulas observed in this study, however, were not compatible with the findings from an earlier report (41). It could be speculated that these conflicting findings are partially due to the differences in the classifications of exposure of interest. For example, in the study of Chen and colleagues, enrollees were recruited with a single diagnostic code for insomnia, which differed from the approach we adopted. Priori study had pointed out while using the administration database, the algorithm requiring at least 3 outpatient service claims, or at least 1 inpatient hospitalization claim during the study period (42). The adopted procedure greatly strengthened the diagnostic validity and minimized possible potential misclassifications of our study.

While our study is the first to investigate the relation between CHMs use and depression risk among patients with insomnia, there are several important limitations. First, when using secondary health care databases, there is always the risk of errors in the coding process. To minimize this bias, we enrolled only persons with new-onset insomnia or depression, and only after the patients had at least three outpatient visits reporting a consistent diagnoses or at least one such inpatient admission. It should also be noted that the NHI of Taiwan randomly reviews the charts and audits medical charges to verify the accuracy of claims. However, the coding approach and data availability were similar between the two groups, and any misclassification bias would have likely been non-differential and toward the null hypothesis, thus, if anything, resulting in an underestimation of the observed effect. Second, the LHID lacks information on social network relationships, family history, personality attributes, laboratory data and education level. Thus, future studies that include these factors would be beneficial in shedding further light on our findings. Third, although continuous use of CHMs in our study indicated a significant benefit, well-designed randomized controlled trials are still needed to confirm the effect of specific medicines. These limitations notwithstanding, this study also had considerable advantages. The database used in this study is representative of the entire Taiwanese population, and the large sample size ensured reliable findings. In addition, the use of a longitudinal cohort study design allowed the robust examination of the causal relationship between high intensity CHMs use and lower risk of depression in people with insomnia.

\section{CONCLUSION}

This is the first large population-based investigation to delineate the impact of CHMs on the risk of depression among patients with insomnia. The findings could serve as references for further studies concerning the influence of CHMs on other medical complications resulting from insomnia. We found that the integration of $\mathrm{CHMs}$ into conventional therapy reduced the subsequent risk of depression by $56 \%$, with greater benefits in those receiving $\mathrm{CHMs}$ for longer than 2 years. Healthcare providers may consider integrating $\mathrm{CHMs}$ into therapeutic care to ameliorate the clinical manifestations of insomnia in their patients.

\section{DATA AVAILABILITY STATEMENT}

The raw data supporting the conclusions of this article will be made available by the authors, without undue reservation.

\section{ETHICS STATEMENT}

The current study involving human participants was reviewed and approved by the Institutional Review Board and Ethics Committee of Buddhist Dalin Tzu Chi Hospital, Taiwan (No. B10004021-3). The ethics committee waived the requirement of written informed consent for participation.

\section{AUTHOR CONTRIBUTIONS}

Y-WC, C-CY, W-JC, and T-YT: study concept and design. M-CLu, T-YT, and H-RG: acquisition of data. T-YT, HL, and H-RG: data analysis. M-CLu and C-CY: project management. M-CLi, HL, T-YT, Y-WC, H-RG, and W-JC: writing. All authors contributed to the article and approved the submitted version.

\section{ACKNOWLEDGMENTS}

This study is based on data from the National Health Insurance Research Database, provided by the Bureau of National Health Insurance (Department of Health) and managed by the National Health Research Institutes. The interpretation and conclusions contained herein do not represent those of the Bureau of National Health Insurance, Department of Health, the National Health Research Institutes, or the study funders. 


\section{REFERENCES}

1. Mai E, Buysse DJ. Insomnia: prevalence, impact, pathogenesis, differential diagnosis, and evaluation. Sleep Med Clin. (2008) 3:167-74. doi: 10.1016/j.jsmc.2008.02.001

2. Parthasarathy S, Vasquez MM, Halonen M, Bootzin R, Quan SF, Martinez FD, et al. Persistent insomnia is associated with mortality risk. Am J Med. (2015) 128:268-75. doi: 10.1016/j.amjmed.2014.10.015

3. Stoller MK. Economic effects of insomnia. Clin Ther. (1994) 16:873-897.

4. Baglioni C, Battagliese G, Feige B, Spiegelhalder K, Nissen C, Voderholzer $\mathrm{U}$, et al. Insomnia as a predictor of depression: a meta-analytic evaluation of longitudinal epidemiological studies. J Affect Disord. (2011) 135:109. doi: 10.1016/j.jad.2011.01.011

5. Cuijpers P, Vogelzangs N, Twisk J, Kleiboer A, Li J, Penninx BW. Comprehensive meta-analysis of excess mortality in depression in the general community versus patients with specific illnesses. Am J Psychiatry. (2014) 171:453-62. doi: 10.1176/appi.ajp.2013.13030325

6. Yeung WF, Chung KF, Ng KY, Yu YM, Zhang SP, Ng BF, et al. Prescription of Chinese herbal medicine in pattern-based traditional Chinese medicine treatment for depression: a systematic review. Evid Based Complement Alternat Med. (2015) 2015:160189. doi: 10.1155/2015/160189

7. FZ Li, Xu B, Shi HY, Zhang T, Song Z, Chen Y, et al. Efficacy and safety of TCM Yangxin Anshen therapy for insomnia: a systematic review and meta-analysis. Medicine. (2020) 99:8. doi: 10.1097/MD.0000000000019330

8. Shi MM, Piao JH, Xu XL, Zhu L, Yang L, Lin FL, et al. Chinese medicines with sedative-hypnotic effects and their active components. Sleep Med Rev. (2016) 29:108-18. doi: 10.1016/j.smrv.2015.10.001

9. Yeung WF, Chung KF, Ng KY, Yu YM, Ziea ETC, Ng BFL, et al. A systematic review on the efficacy, safety and types of Chinese herbal medicine for depression. J Psychiatr Res. (2014) 57:165-75. doi: 10.1016/j.jpsychires.2014.05.016

10. Wang YS, Shen CY, Jiang JG. Antidepressant active ingredients from herbs and nutraceuticals used in TCM: pharmacological mechanisms and prospects for drug discovery. Pharmacol Res. (2019) 150:104520. doi: 10.1016/j.phrs.2019.104520

11. The National Health Research Institutes. National Health Insurance Research Database. LHID 2000 (2002). Available online at: https://nhird.nhri.org.tw/ (accessed June 15, 2017).

12. HH Li, Livneh H, Yeh CC, Guo HR, Lai NS, Lu MC, et al. Association between use of Chinese herbal medicine and depression risk in patients with rheumatoid arthritis: a nationwide retrospective cohort study. Int J Rheum Dis. (2019) 22:986-94. doi: 10.1111/1756-185X.13571

13. Tsai TY, CY Li, Livneh H, Lin IH, Lu MC, Yeh CC. Decreased risk of stroke in patients receiving traditional Chinese medicine for vertigo: a population-based cohort study. J Ethnopharmacol. (2016) 184:13843. doi: 10.1016/j.jep.2016.03.008

14. Shariff SZ, Cuerden MS, Jain AK, Garg AX. The secret of immortal time bias in epidemiologic studies. J Am Soc Nephrol. (2008) 19:8413. doi: 10.1681/ASN.2007121354

15. Liu CY, Hung YT, Chuang YL, Chen YJ, Weng WS, Liu JS. Incorporating development stratification of Taiwan townships into sampling design of large scale health interview survey. J Health Manage. (2006) 4:1-22. doi: 10.29805/JHM.200606.0001

16. Deyo RA, Cherkin DC, Ciol MA. Adapting a clinical comorbidity index for use with ICD-9-CM administrative databases. J Clin Epidemiol. (1992) 45:613-9. doi: 10.1016/0895-4356(92)90133-8

17. Gooneratne NS. Complementary and alternative medicine for sleep disturbances in older adults. Clin Geriatr Med. (2008) 24:121-38. doi: 10.1016/j.cger.2007.08.002

18. Ni X, Shergis JL, Guo X, Zhang AL, Li Y, Lu C. Updated clinical evidence of Chinese herbal medicine for insomnia: a systematic review and meta-analysis of randomized controlled trials. Sleep Med. (2015) 16:146281. doi: 10.1016/j.sleep.2015.08.012

19. Zhang Y, Wang ZZ, Sun HM, Li P, YF Li, Chen NH. Systematic review of traditional chinese medicine for depression in Parkinson's disease. Am J Chin Med. (2014) 42:1035-51. doi: 10.1142/S0192415X14500657

20. Buysse DJ, Reynolds CF, Kupfer DJ, Thorpy MJ, Bixler E, Manfredi, R, et al. Clinical diagnoses in 216 insomnia patients using the international classification of sleep disorders (ICSD), DSM-IV and ICD-10 categories: a report from the APA/NIMH DSM-IV field trial. Sleep. (1994) 17:6307. doi: 10.1093/sleep/17.7.630

21. Shu H, Arita H, Hayashida M, Zhang L, An K, Huang W, et al. Anti-hypersensitivity effects of Shu-jing-huo-xue-tang, a Chinese herbal medicine, in CCI-neuropathic rats. J Ethnopharmacol. (2010) 131:46470. doi: 10.1016/j.jep.2010.07.004

22. Cottingham C, Wang Q. Alpha2 adrenergic receptor dysregulation in depressive disorders: implications for the neurobiology of depression and antidepressant therapy. Neurosci Biobehav Rev. (2012) 36:221425. doi: 10.1016/j.neubiorev.2012.07.011

23. Minaei A, Haghdoost-Yazdi H. Dexmedetomidine attenuates the induction and reverses the progress of 6-hydroxydopamine- induced parkinsonism; involvement of KATP channels, alpha 2 adrenoceptors and anti-inflammatory mechanisms. Toxicol Appl Pharmacol. (2019) 382:114743. doi: 10.1016/j.taap.2019.114743

24. Wang CL, Wang LY, Wang JX, Zhu YL, Zhao DP, Wu L, et al. Effects of albiflorin, paeoniflorin on the behavior and hypothalamic-pituitary-adrenal axis in depressed rats modelized by chronic restraint stress stimulation combined with isolation. China J Tradit Chinese Med Pharm. (2017) 7:3121-5.

25. Cheng J, Dong S, Yi L, Geng D, Liu Q. Magnolol abrogates chronic mild stress-induced depressive-like behaviors by inhibiting neuroinflammation and oxidative stress in the prefrontal cortex of mice. Int Immunopharmacol. (2018) 59:61-7. doi: 10.1016/j.intimp.2018.03.031

26. Wu M, Liang S, Ma L, Han Y, Zhang X, Xu C. Effects of delayed puerarin treatment in long-term neurological outcomes of focal ischemic stroke in rats. Indian J Pharmacol. (2014) 46:157-60. doi: 10.4103/0253-7613.129305

27. Lopresti AL. Salvia (Sage): a review of its potential cognitiveenhancing and protective effects. Drugs $R$ D. (2017) 17:5364. doi: 10.1007/s40268-016-0157-5

28. Ma S, Zhang D, Lou H, Sun L, Ji J. Evaluation of the antiinflammatory activities of tanshinones isolated from Salvia miltiorrhiza var. alba roots in THP-1 macrophages. J Ethnopharmacol. (2016) 188:193-9. doi: 10.1016/j.jep.2016.05.018

29. Dowlati Y, Herrmann N, Swardfager W, Liu H, Sham L, Reim EK, et al. A meta-analysis of cytokines in major depression. Biol Psychiatry. (2010) 67:446-57. doi: 10.1016/j.biopsych.2009.09.033

30. Laveti D, Kumar M, Hemalatha R, Sistla R, Naidu VG, Talla V, et al. Antiinflammatory treatments for chronic diseases: a review. Inflamm Allergy Drug Targets. (2013) 12:349-61. doi: 10.2174/18715281113129990053

31. Piao HZ, Jin SA, Chun HS, Lee JC, Kim WK. Neuroprotective effect of wogonin: potential roles of inflammatory cytokines. Arch Pharm Res. (2004) 27:930-6. doi: 10.1007/BF02975846

32. Monisha BA, Kumar N, Tiku AB. Emodin and its role in chronic diseases. $A d v$ Exp Med Biol. (2016) 928:47-73. doi: 10.1007/978-3-319-41334-1_3

33. Yi PF, Wu YC, Dong HB, Guo Y, Wei Q, Zhang C, et al. Peimine impairs pro-inflammatory cytokine secretion through the inhibition of the activation of NF-kappaB and MAPK in LPS-induced RAW264.7 macrophages. Immunopharmacol Immunotoxicol. (2013) 35:567-72. doi: 10.3109/08923973.2013.822508

34. Lim HS, Kim YJ, Kim BY, Park G, Jeong SJ. The anti-neuroinflammatory activity of tectorigenin pretreatment via downregulated NFkappaB and ERK/JNK pathways in BV-2 microglial and microglia inactivation in mice with lipopolysaccharide. Front Pharmacol. (2018) 9:462. doi: 10.3389/fphar.2018.00462

35. YC Li, Shen JD, Li J, Wang R, Jiao S, Yi LT. Chronic treatment with baicalin prevents the chronic mild stress-induced depressive-like behavior: involving the inhibition of cyclooxygenase-2 in rat brain. Prog Neuropsychopharmacol Biol Psychiatry. (2013) 40:138-43. doi: 10.1016/j.pnpbp.2012.09.007

36. Yu HY, Yin ZJ, Yang SJ, Ma SP. Baicalin reverse AMPA receptor expression and neuron apoptosis in chronic unpredictable mild stress rats. Biochem Biophys Res Commun. (2014) 451:467-72. doi: 10.1016/j.bbrc.2014.07.041

37. Acheson A, Conover JC, Fandl JP, DeChiara TM, Russell M., Thadani A, et al. A BDNF autocrine loop in adult sensory neurons prevents cell death. Nature. (1995) 374:450-3. doi: 10.1038/374450a0

38. Yu H, Chen ZY. The role of BDNF in depression on the basis of its location in the neural circuitry. Acta Pharmacol Sin. (2011) 32:311. doi: 10.1038/aps.2010.184 
39. Seidlova-Wuttke D, Hesse O, Jarry H, Rimoldi G, Thelen P, Christoffel $\mathrm{V}$, et al. Belamcanda chinensis and the thereof purified tectorigenin have selective estrogen receptor modulator activities. Phytomedicine. (2004) 11:392-403. doi: 10.1016/j.phymed.2004.01.003

40. Gong P, Deng F, Zhang W, Ji J, Liu J, Sun Y, et al. Tectorigenin attenuates the MPP(+)-induced SH-SY5Y cell damage, indicating a potential beneficial role in Parkinson's disease by oxidative stress inhibition. Exp Ther Med. (2017) 14:4431-7. doi: 10.3892/etm.2017.5049

41. Chen FP, Jong MS, Chen YC, Kung YY, Chen TJ, Chen FJ, et al. Prescriptions of Chinese herbal medicines for insomnia in Taiwan during 2002. Evid Based Complementary Altern Med. (2011) 2011:236341. doi: 10.1093/ecam/ nep018

42. Scherrer JF, Virgo KS, Zeringue A, Bucholz KK, Jacob T, Johnson RG, et al. Depression increases risk of incident myocardial infarction among veterans administration patients with rheumatoid arthritis. Gen Hosp Psychiatry. (2009) 31:353-9. doi: 10.1016/j.genhosppsych.2009.04.001

Conflict of Interest: The authors declare that the research was conducted in the absence of any commercial or financial relationships that could be construed as a potential conflict of interest.

Copyright ( 2021 Chiao, Livneh, Guo, Chen, Lu, Lin, Yeh and Tsai. This is an open-access article distributed under the terms of the Creative Commons Attribution License (CC BY). The use, distribution or reproduction in other forums is permitted, provided the original author(s) and the copyright owner(s) are credited and that the original publication in this journal is cited, in accordance with accepted academic practice. No use, distribution or reproduction is permitted which does not comply with these terms. 